\title{
Prevalence of chronic health conditions and hospital utilization in adults with spinal cord injury: an analysis of self-report and South Carolina administrative billing data
}

\author{
Nicole D. DiPiro ${ }^{1} \cdot$ David Murday $^{2} \cdot$ Elizabeth H. Corley $^{2} \cdot$ James S. Krause $^{1}$ \\ Received: 31 January 2018 / Revised: 22 June 2018 / Accepted: 25 June 2018 / Published online: 8 August 2018 \\ (c) International Spinal Cord Society 2018
}

\begin{abstract}
Study design Retrospective analysis of self-report and administrative billing data.

Objectives (1) Identify the self-reported prevalence of seven chronic health conditions (CHCs) in adults with chronic, traumatic spinal cord injury (SCI), (2) Examine the relationships between the presence of CHC with future hospital admissions and total number of inpatient days and (3) identify predictors of utilization.

Setting Data were collected from participants living in and utilizing hospitals in South Carolina, USA.

Methods Participants were identified through the South Carolina SCI Surveillance System Registry. Between 2010 and 2013, 963 adults ( $>18$ years old) with chronic ( $>1$-year), traumatic SCI completed self-report assessments (SRAs); this analysis includes data from 787 individuals. The presence/absence of the seven CHC was assessed using self-report data. Administrative billing data were used to assess hospital utilization in non-federal, South Carolina hospitals in the year following the SRA.

Results In all, $40.5 \%$ reported no CHC; $23.4 \%$ reported one $\mathrm{CHC}$ and $36.1 \%$ reported having two or more CHC. The most commonly reported CHCs were hypertension (43.1\%), high cholesterol (32.2\%) and diabetes (15.8\%). In total, 59\% had at least one hospital admission in the year following the SRA (mean 3 \pm 5 ; range $0-45$; median $=1$ ). The mean total inpatient days was $15.7 \pm 43$ days (range $0-365$; median $=1$ ). Predictors of hospital admission included CHC, pressure sores, education, prior hospitalization and injury severity. With the exception of CHC, each was also associated with total number of inpatient days.
\end{abstract}

Conclusions CHC are prevalent and associated with hospital admissions in adults with chronic, traumatic SCI.

\section{Introduction}

Traumatic spinal cord injury (SCI) often results in physical disability and an elevated risk of long-term health complications, including chronic health conditions (CHCs) [1-4]. $\mathrm{CHC}$ (e.g., heart disease, hypertension, diabetes and cancer) are commonly defined as conditions lasting for an extended period of time (>12 months), which develop slowly, require ongoing medical attention and limit daily activities

James S. Krause

krause@musc.edu

1 College of Health Professions, Medical University of South Carolina, Charleston, SC, USA

2 Arnold School of Public Health, University of South Carolina, Columbia, SC, USA
[5, 6]. CHC can be distinguished from secondary health conditions (SHCs), which arise as a result of SCI (e.g., pressure sores) [1, 2, 4], as $\mathrm{CHC}$ include pre-existing, typically progressive diseases and conditions not considered a direct result of disability $[4,5]$.

Studies suggest that individuals with SCI may experience an accelerated onset of CHC usually associated with the aging process, and at higher rates than age-matched individuals without disability $[7,8]$. In particular, increased risk and prevalence of cardiovascular disease [9-11], heart attack [12, 13], stroke [9, 10, 14], diabetes [12, 15, 16], hypertension [12] and dyslipidemia [12] have been observed in both chronic and acute SCI [17]. While a number of studies have assessed the incidence and prevalence of specific $\mathrm{CHC}$, rarely has the occurrence of multiple chronic conditions (MCCs) been studied in individuals with SCI $[3,18]$. The presence of MCC, defined as two or more $\mathrm{CHC}$, has been found to complicate medical 
care, increase the risk and duration of hospitalization, and raise the risk of mortality [6,19-21]. Owing to the profound health consequences, health care utilization and costs associated with having MCC, there has been increased emphasis on studying MCC in the general population in recent years [22-24]. However, few studies have addressed comorbid CHC in individuals with chronic SCI, and limited data exist regarding associated hospital utilization in the United States.

One recently published large-scale study of adults with chronic SCI $(n=1678)$ reported the lifetime prevalence of concurrent $\mathrm{CHC}$ [3]. Nearly half of the sample reported at least one $\mathrm{CHC}$, and $23.2 \%$ reported having MCC. Another study recently documented the prevalence and incidence of five CHC in individuals with long-term disability, including 414 participants with SCI [18]. The study found that having one or more $\mathrm{CHC}$ is a significant predictor of incidence of additional CHC [18]. Although high prevalence rates were reported for each of the $\mathrm{CHC}$ and $\mathrm{MCC}$, the findings were not broken down by disability type, thus it is not possible to report on those with SCI.

The current literature identifies a need for the study of comorbid CHC in individuals with SCI and the associations between $\mathrm{CHC}$ and health care utilization. High rates of hospital admissions are commonly observed after SCI, and hospitalizations largely contribute to the high costs of living [25-28]. Previous studies have identified risk factors for and causes of re-hospitalization after SCI [25, 29-33]. The most commonly reported causes are SHC including urinary tract infections, pressure sores, pneumonia, pain and depression [29, 34-37]. The relationships between CHC and hospital utilization have not been adequately studied. Additionally, although multimorbidity has been associated with "inappropriate health care utilization" [30] in individuals with SCI, the relationship between MCC and hospital admissions or total number of days hospitalized has not been assessed. Considering the increased need for medical care and risk of hospitalization associated with the presence of $\mathrm{CHC}$ and MCC in the general population, it is reasonable to hypothesize similar relationships will be observed in those with SCI. Knowledge of this relationship is crucial to understanding the types of prevention needed to attenuate future risks and burden of hospitalization.

Our purpose was to (1) identify the prevalence of seven $\mathrm{CHC}$, including diabetes, heart attack, coronary heart disease, stroke, hypertension, high cholesterol and cancer in adults with chronic SCI using a self-report assessment (SRA), (2) to merge this with administrative billing data to examine the relationships between the presence of $\mathrm{CHC}$ and hospital utilization (hospital admissions and total number of inpatient days) in the 12 months after the SRA and (3) identify predictors of utilization.

\section{Methods}

A detailed description of the self-report and administrative data collection procedures has been previously published [28]. All study procedures were approved by an institutional review board.

\section{Participants}

Participants were identified through the South Carolina SCI Surveillance Registry (SCSCISR), a populationbased registry of SCI occurring in the state of South Carolina. All non-federal hospitals are mandated to report discharge data on hospitalizations involving SCI to the South Carolina Office of Revenue and Fiscal Affairs, Health, and Demographics (RFAHD) through the uniform billing discharge data (UB-04). The SCSCISR does not include military or veteran hospitals and, therefore, represents the civilian population. Duplicate admissions are eliminated using personal identifiers. Individuals who have moved out of state are excluded, as well as cases presenting as late effects of SCI (i.e., not incident cases).

From the SCSCISR, 3132 individuals with a hospital discharge from 1998 to 2012 were recruited. There were 963 respondents to the SRA $(30.7 \%)$. The reasons for nonresponse included: deceased $(n=199)$, refusal to participate $(n=706)$ and lost/unable to reach $(n=1264)$. Inclusion criteria were: $(1) \geq 18$ years of age, $(2) \geq 1$-year post-injury and (3) traumatic SCI with residual effects (i.e., not complete recovery). Preliminary analyses have shown individuals with SCI from certain counties bordering other states are likely to seek care in those states. Respondents from those counties $(n=153)$ were excluded because South Carolina administrative billing data would not capture all of their hospitalizations. Respondents surveyed after 1 January $2016(n=23)$ were excluded since 2017 utilization data were not available. Of the 963 respondents, 176 were excluded from the current analysis. The current analysis includes 787 participants.

\section{Data collection procedures}

\section{Self-report assessments}

SRA were collected by mail between 2010 and 2013. Prospective participants were sent a letter describing the study and notifying them of forthcoming materials. Individuals who did not respond to the initial request received second mailings and follow-up phone calls. If requested by the participant, a third mailing was sent. Participants received $\$ 50$ remuneration. 


\section{Administrative billing data}

The administrative billing data source is limited to nonfederal hospitals in South Carolina. We controlled for outmigration (receiving treatment in a different state where records are not available) as previously detailed, by eliminating those participants from counties where outmigration for hospitalizations was most likely to occur [28]. Data on number of hospital admissions were acquired through the RFAHD, which receives medical record and charges data from South Carolina hospitals at least quarterly. We examined records of hospitalizations in the 12 months after the SRA date. General inpatient, specialty hospital inpatient (rehabilitation, psychiatric) and long-term acute care hospitalizations were included in the calculation of hospitalizations. All types of hospital admissions (including longterm acute and specialty) were used. All but one SRA respondent were matched to RFAHD administrative records.

The outcome variables abstracted from the administrative billing records were: (1) inpatient hospital admissions and (2) total number of days spent in hospital care. In the 12 months after responding to the SRA, inpatient admissions ranged from 0 to 45 admissions, and total inpatient days ranged from 0 to 365 days in the year following the SRA.

\section{Measures}

Participants completed a composite SRA comprised of multiple measures. The SRA included sociodemographic and injury variables, as well as variables assessing health conditions and prior utilization. Participants were asked age, gender, race (collapsed to white/non-white), highest level of education (six ordinal categories), annual household income from all sources ( $>\$ 25 \mathrm{~K}, \$ 25-75 \mathrm{~K}$ and $>\$ 75 \mathrm{~K}$ ), community type (urban or rural) and relationship status (dichotomized as married/couple and single). Injury characteristics included time since injury (years) and injury severity, categorized into five groups: (1) $\mathrm{C} 1-\mathrm{C} 4$, non-ambulatory, (2) C5-C8, non-ambulatory, (3) non-cervical, non-ambulatory, (4) ambulatory, regardless of injury level $(<1000$ feet) and (5) ambulatory, able to walk $>1000$ feet. A similar classification scheme, with only four levels of injury severity has been frequently used in studies of health and mortality in individuals with SCI $[3,28]$. This classification scheme serves as a proxy measure for the American Spinal Injury Association (ASIA) Impairment Scale (AIS A-D). Due to the large number of ambulatory participants in the current sample, an additional level of ambulatory status (walk > 1000 feet) was included.

The presence/absence of seven CHC was assessed using standardized questions from the Behavioral Risk Factor
Surveillance System (BRFSS) available at the time of the SRA development. Participants were asked, "Has a doctor, nurse, or other health professional ever told you that you had [name of condition]?" The conditions were presented as follows: (1) diabetes (not including gestational), (2) heart attack (also called a myocardial infarction), (3) angina or coronary heart disease, (4) a stroke, (5) high blood pressure (hypertension, not including during pregnancy), (6) high cholesterol or (7) cancer. The total number of $\mathrm{CHC}$ was summed (0-7). Individuals reporting two or more CHCs were classified as having MCC.

Additionally, the SRA included a series of questions on pressure sores, defined as "open sores in pressure areas, such as your tailbone, ischium, heel, and elbows, which are usually caused by pressure but may also be caused by friction or shearing (rubbing), moisture, burns, or falls." Participants reported the presence/absence of a current pressure/skin sore. Pressure sores are a serious SHC and have been previously reported to increase the risk of hospitalization and lengths of stay; therefore, they were included in the analysis as a statistical control [37, 38].

Self-reported health care access and prior utilization were also assessed and included as predictors. Participants were asked if there was 'a place or places where they usually go when sick or in need of health advice.' They were asked if they had 'missed or postponed needed medical care due to lack of money, insurance, or transportation.' They were asked to report, "In the past year (12 months), how many times (if any) have you been hospitalized for any reason?" The variable ranged from ' 0 to $10+$ ' hospitalizations.

\section{Statistical analyses}

All analyses were completed with SAS Version 9.4 (SAS Institute). Descriptive statistics, mean (SD) or $n(\%)$, are presented. Bivariate analyses were conducted and multiple regression models were tested. Given the many potential predictor variables and the large number of observations missing data for one or more of those variables, nonsignificant predictor variables were dropped from the models unless they improved goodness of fit as measured by Akaike information criterion (AIC). This maximized the number of observations included in each analysis; 581 observations were analyzed. The incidence rate ratios (IRRs) and 95\% confidence intervals are presented.

\section{Results}

\section{Descriptive}

The current study included 787 participants with chronic, traumatic SCI; $72.2 \%$ were male, $59.2 \%$ were white and the 
Table 1 Participant characteristics $(n=787)$

\begin{tabular}{|c|c|}
\hline & Mean $(\mathrm{SD})$ \\
\hline Age & $49.7(16.7)$ \\
\hline Time since injury & $6.8(8.8)$ \\
\hline \multicolumn{2}{|l|}{$n(\%)$} \\
\hline Gender (male) & $568(72.2)$ \\
\hline Race/ethnicity (white) & $465(59.2)$ \\
\hline \multicolumn{2}{|l|}{ Education } \\
\hline No high school & $175(22.7)$ \\
\hline High school & $289(37.5)$ \\
\hline Associates/vocational/bus & $207(26.9)$ \\
\hline Bachelors & $57(7.4)$ \\
\hline Masters & $28(3.6)$ \\
\hline Doctorate & $14(1.8)$ \\
\hline \multicolumn{2}{|l|}{ Income } \\
\hline$<\$ 25 \mathrm{~K}$ & $466(62.4)$ \\
\hline$\$ 25-75 \mathrm{~K}$ & $199(26.6)$ \\
\hline$>\$ 75 \mathrm{~K}$ & $82(11.0)$ \\
\hline Community type (urban) & $554(70.4)$ \\
\hline Relationship status (single) & $409(52.7)$ \\
\hline Usual place of care (yes) & $707(91.2)$ \\
\hline Missed care (no) & $449(58.2)$ \\
\hline \multicolumn{2}{|l|}{ General health } \\
\hline Poor & $69(8.8)$ \\
\hline Fair & $291(37.1)$ \\
\hline Good & $289(36.9)$ \\
\hline Very good & $105(13.4)$ \\
\hline Excellent & $30(3.8)$ \\
\hline Current pressure sore (yes) & $134(17.5)$ \\
\hline \multicolumn{2}{|l|}{ SRA prior admissions } \\
\hline None & $453(58.2)$ \\
\hline $1-4$ & $297(38.1)$ \\
\hline $5-9$ & $21(2.7)$ \\
\hline $10+$ & $8(1.0)$ \\
\hline \multicolumn{2}{|l|}{ Injury severity } \\
\hline $\mathrm{C} 1-4$, non-ambulatory & $41(6.2)$ \\
\hline C5-8, non-ambulatory & $77(11.7)$ \\
\hline Non-cervical, non-ambulatory & $119(18.0)$ \\
\hline Ambulatory & $212(32.1)$ \\
\hline Ambulatory, walk 1000 feet & $212(32.1)$ \\
\hline
\end{tabular}

average age was 49.7 years (Table 1). Seventy-seven percent of participants had a minimum of high school education. Forty-four percent reported household income of $\$ 20,000$ or more.

Of participants, $40.5 \% \quad(n=317)$ reported no $\mathrm{CHC}$; $23.4 \%(n=183)$ reported having only one $\mathrm{CHC}$ and $36.1 \%$ ( $n=283$ ) reported having MCC (two or more CHC). The three most commonly reported CHCs were hypertension
Table 2 Self-reported chronic health conditions (CHCs)

\begin{tabular}{lll}
\hline Chronic health conditions & Frequency & Percent \\
\hline Hypertension & 355 & 43.1 \\
High cholesterol & 250 & 32.2 \\
Diabetes & 123 & 15.8 \\
Cancer & 73 & 9.4 \\
Stroke & 67 & 8.7 \\
Angina or coronary heart disease & 64 & 8.2 \\
Heart attack & 59 & 7.6 \\
Multiple chronic conditions $(\geq 2 \mathrm{CHC})$ & 238 & 36.1 \\
\hline
\end{tabular}

(43.1\%), high cholesterol (32.2\%) and diabetes (15.8\%). Less than $10 \%$ of participants reported having had a heart attack, angina, stroke or cancer (Table 2).

Fifty-nine percent of participants had at least one hospital admission in the 12 months following the SRA. A total of 2366 hospital admissions were reported in the same time frame. On average, participants reported $3 \pm 5$ admissions (range $0-45$, median $=1$ ). Forty-one percent of participants had zero inpatient admissions, $21.7 \%$ had $1-5$ admissions, 9.5\% had 6-10 admissions and $7.4 \%$ had 10 or more hospital admissions in the year following the SRA. The mean total inpatient days in the hospital was $15.7 \pm 43$ days (range $0-365$, median $=1$ ). Of those hospitalized, $34.3 \%$ of participants spent a total of 1-7 days in the hospital, $6.6 \%$ spent $8-14$ days, $5.1 \%$ spent $15-29$ days and $12.5 \%$ spent 30 or more days in the hospital.

\section{Regression}

There were five significant predictors of the number of hospital admissions (Table 3). Bivariate results and results from the final multivariate regression are presented. In the final model, the presence of a pressure sore at or near the time of the assessment, greater number of $\mathrm{CHC}$, more education and more self-reported prior hospitalizations were associated with more admissions. For example, for every additional CHC (summed 0-7), there is a $12 \%$ increase in the rate of hospital admissions. More severe SCI was also associated with more admissions (injury severity: higher score = less severe). Income, race, gender, relationship status, usual place of care and missed care were not predictive of hospital admissions.

In the final model, there were four significant predictors of inpatient hospital days, including the presence of a pressure sore at or near the time of the SRA, more education, more reported prior hospitalizations and more severe SCI (Table 3). The number of CHC was not significantly associated with the total number of inpatient hospital days. None of the other factors was significant. 
Table 3 Predictors of subsequent hospital admissions and total days admitted $(n=581)$

\begin{tabular}{|c|c|c|c|c|c|c|c|c|c|c|c|c|}
\hline & \multicolumn{6}{|c|}{ Number of hospital admissions } & \multicolumn{6}{|c|}{ Total days in the hospital } \\
\hline & \multicolumn{2}{|c|}{ Unadjusted } & \multicolumn{4}{|c|}{ Final multivariate regression } & \multicolumn{2}{|c|}{ Unadjusted } & \multicolumn{4}{|c|}{ Final multivariate regression } \\
\hline & IRR & $p$ & IRR & $95 \%$ & & $p$ & IRR & $p$ & IRR & $95 \%$ & & $p$ \\
\hline Urban (rural) & 0.96 & 0.8031 & & & & & 0.98 & 0.9204 & & & & \\
\hline General health & 0.90 & 0.1829 & & & & & 0.97 & 0.8128 & & & & \\
\hline Able to walk (no) & 0.42 & $<0.0001$ & & & & & 0.22 & $<0.0001$ & & & & \\
\hline Age & 1.00 & 0.8321 & & & & & 0.99 & 0.0295 & & & & \\
\hline Race (white) & 1.15 & 0.3115 & 1.17 & 0.88 & 1.54 & 0.2808 & 1.11 & 0.5801 & & & & \\
\hline Gender (male) & 1.22 & 0.1800 & 1.29 & 0.99 & 1.69 & 0.0638 & 0.94 & 0.7758 & 1.35 & 0.90 & 2.02 & 0.1464 \\
\hline Injury severity & 0.69 & $<0.0001$ & 0.79 & 0.70 & 0.89 & 0.0001 & 0.49 & $<0.0001$ & 0.59 & 0.49 & 0.70 & $<0.0001$ \\
\hline Income & 1.02 & 0.3720 & 1.02 & 0.96 & 1.09 & 0.5443 & 0.99 & 0.7202 & 0.98 & 0.91 & 1.07 & 0.6837 \\
\hline Education & 1.14 & 0.0254 & 1.21 & 1.07 & 1.37 & 0.0021 & 1.12 & 0.2106 & 1.44 & 1.19 & 1.75 & 0.0002 \\
\hline Relationship status (no sig. other) & 0.89 & 0.4074 & 0.87 & 0.66 & 1.14 & 0.3149 & 0.69 & 0.0554 & 0.85 & 0.57 & 1.27 & 0.4313 \\
\hline Usual place of care (no) & 1.79 & $\mathbf{0 . 0 2 7 7}$ & 1.37 & 0.83 & 2.28 & 0.2206 & 1.40 & 0.3580 & 0.78 & 0.39 & 1.57 & 0.4858 \\
\hline Missed care & 0.81 & 0.0285 & 0.88 & 0.73 & 1.07 & 0.2160 & 0.95 & 0.6983 & 0.91 & 0.68 & 1.22 & 0.5427 \\
\hline Current pressure sore (no) & 2.54 & $<0.0001$ & 2.00 & 1.44 & 2.78 & $<0.0001$ & 4.11 & $<0.0001$ & 2.84 & 1.73 & 4.64 & $<0.0001$ \\
\hline Chronic conditions & 1.07 & 0.1999 & 1.12 & 1.02 & 1.23 & 0.0235 & 0.93 & 0.3188 & 1.05 & 0.91 & 1.21 & 0.4807 \\
\hline SRA prior admission & 1.30 & $<0.0001$ & 1.18 & 1.08 & 1.28 & 0.0001 & 1.38 & $<0.0001$ & 1.21 & 1.06 & 1.39 & 0.0051 \\
\hline
\end{tabular}

IRR incidence rate ratio, $C I$ confidence interval, $S R A$ self-reported assessment

\section{Discussion}

In the United States, roughly $50 \%$ of the adult population has one $\mathrm{CHC}$, and $31.5 \%$ have MCC $[6,39]$. Considering the profound health consequences of $\mathrm{CHC}$ and increased rates and lengths of hospitalization related to $\mathrm{CHC}$ and MCC in the general population, we sought to examine the relationships between the presence of $\mathrm{CHC}$ and hospital utilization in individuals with chronic, traumatic SCI. We used self-report responses to predict those individuals with a SCI who are at greatest risk for increased hospital inpatient admissions and total days hospitalized per year. We found that having a greater number of $\mathrm{CHC}$ was associated with more hospital admissions but not the total days hospitalized.

We examined the rates of seven $\mathrm{CHC}$ and MCC. Overall, compared with publicly available South Carolina (general population) BRFSS prevalence and trends data [40] that corresponds to the time frame of our SRA (2010-2013), our participants reported higher rates of hypertension $(43.1 \%$ vs. $38.4 \%)$, diabetes $(15.8 \%$ vs. $12.5 \%)$, stroke $(8.7 \%$ vs. $3.8 \%)$, angina or coronary heart disease $(8.2 \%$ vs. $5.1 \%)$, heart attack $(7.6 \%$ vs. $5.2 \%)$ and lower rates of high cholesterol (32.3\% vs. $42.6 \%)$. We found a larger percentage of participants reported hypertension than expected based on previous studies in individuals with SCI (6-29\%) [2,3]. The reported rates of diabetes and coronary heart disease are within the ranges previously presented in the literature, $5-22 \%$ and
3.6-24\%, respectively [2]. Compared with prevalence rates from a clinical cohort primarily from the state of Georgia [3], our population-based cohort had a slightly greater percentage of participants reporting each of the seven CHC. Among participants, 36.1\% reported having MCC, higher than rates reported in an earlier study of individuals with SCI (23.3\%) [3], and higher than estimates for the general population.

In terms of re-hospitalization and lengths of stay, data from the National Spinal Cord Injury Database suggest roughly $30 \%$ of individuals with SCI are re-hospitalized at least once in any given year after injury, with an average length of stay of 22 days [41]. Although the SCI Model Systems data do indicate causes of re-hospitalization, they do not identify specific CHC. Further, although studies have examined risk factors for hospitalization, data regarding the association of $\mathrm{CHC}$ and hospital utilization are lacking. Previous studies of hospital utilization have found that decreased physical health, increased injury severity and increased time since injury are associated with increased hospitalization [28, 42]. In general, findings are mixed regarding race and education as predictors of hospitalization after SCI [28, 42].

Our findings support injury severity as a predictor and highlight the importance of $\mathrm{CHC}$. In line with findings from the general population, we found that increasing number of $\mathrm{CHC}$ (having more $\mathrm{CHC}$, i.e., $\mathrm{MCC}$ ) was associated with an increased number of hospital admissions [22, 43]; however, CHCs were not associated with 
the total number of inpatient days in the hospital. Prior hospitalization, greater severity of SCI, current/recent pressure sore and more education were significantly associated with both hospital admissions and total number of days spent in the hospital. Contrary to previous selfreport findings [42], more education was associated with greater number of hospital admissions and days in the hospital. Although only included as a statistical control, our findings confirm the risks of pressure sores for hospital admissions and highlight that pressure sores are associated with a greater number of days spent in the hospital per year [37, 38].

\section{Methodologic considerations}

There are important methodologic considerations to acknowledge when evaluating the results. First, this study is relatively unique in combining self-report and objective administrative billing data. However, as the predictor and CHC variables came from a SRA, there is potential for recall bias. This is a limitation with all self-report data. Second, we had a $30.7 \%$ response rate for the SRA. Followup studies of population-based cohorts lack a natural point of contact that is typically present in studies of clinical cohorts in which the individual has received services from a particular institution and is not surprised by further contact. Third, the patient identifiable administrative billing data were limited to visits occurring within South Carolina; we did not capture South Carolina residents who may have sought care across state lines. Fourth, it is important to highlight that the SCSCISR data are population-based and, therefore, has greater generalizability to the full SCI population when compared with data obtained from clinical service settings, particularly those with specialty services, including the SCI Model Systems. Individuals with less severe SCI and those who have an inability to pay may fall through the cracks of clinical settings and be less well represented in research. Finally, our measurement of CHC was defined by whether the condition had ever occurred, modeled on items used in the general population BRFSS. The incidence of CHC (prior to or after SCI) was not assessed. Since the development of our SRA, additional chronic conditions have been added to the BRFSS.

\section{Future directions}

This study is novel and innovative in the linkage of selfreported $\mathrm{CHC}$ with subsequent hospital utilization. Overall, we observed relatively high rates of $\mathrm{CHC}$ and MCC in our predominantly middle-aged cohort, and we can expect the prevalence rates to rise as the population ages. It is imperative that future research and care shift focus toward concurrent $\mathrm{CHC}$ and managing clusters of $\mathrm{CHC}$ to prevent adverse outcomes, including costly hospitalizations and mortality. The health and wellness of adults with long-term $\mathrm{SCI}$ is contingent upon increased awareness of the prevalence and risks of $\mathrm{CHC}$, coordinated care, and effective prevention and treatment efforts.

Our results highlight the associations between increasing number of CHC (i.e., MCC) and hospital admissions. These findings may alert consumers to the risks of having MCC and may allow care managers to intervene and possibly reduce the need for hospitalizations and days spent in the hospital. Continued study of the relationship between $\mathrm{CHC}$ and hospital utilization, considering additional $\mathrm{CHC}$ and controlling for the presence of SHC, is necessary to better understand these relationships.

Although we observed a substantial number of hospital admissions, we have yet to analyze the associated costs. Utilization of health services is a critical topic in understanding costs and patterns of care after SCI, and continued study is warranted. In the USA, the costs associated with CHC exceed $\$ 100$ billion annually, and a disproportionate, $71 \%$ share of the nation's health care costs is attributed to MCC [39]. Future analysis of cost data is necessary to identify the costs associated with CHC hospitalizations and prevent costly outcomes in individuals with SCI.

\section{Conclusion}

CHCs were prevalent. More than a third of the current study sample reported having MCC. As the number of CHC increased, so did hospital admissions. However, CHCs were not associated with the total number of inpatient days. Continued large-scale, population-based study is necessary to understand the incidence, prevalence and clustering of $\mathrm{CHC}$, and the associations with hospital utilization and cost.

Author contributions NDD was responsible for leading the manuscript, drafting of the research questions and revising the manuscript. $\mathrm{DM}$ was responsible for analyzing and interpreting the data. He contributed to revising the manuscript. $\mathrm{BC}$ was responsible for analyzing and interpreting the data. She contributed to revising the manuscript. JSK is the senior investigator and Principal Investigator of the research program. He was responsible for designing and directing the project and served as a mentor to the lead author, as well as being responsible for revising the manuscript and approving the final version.

Funding The contents of this publication were developed under grants from the National Institute on Disability, Independent Living, and Rehabilitation Research (NIDILRR grant numbers 90IF0119 and 90RT5003), and the South Carolina Spinal Cord Injury Research Fund (SCSCIRF) grant \# SCIRF 09-001. NIDILRR is a Center within the Administration for Community Living (ACL), Department of Health and Human Services (HHS). The contents of this publication do not necessarily represent the policy of NIDILRR, ACL, HHS, SCSCIRF and you should not assume endorsement by the Federal Government or the state of South Carolina. 


\section{Compliance with ethical standards}

Conflict of interest The authors declare that they have no conflict of interest.

Statement of ethics We certify that all applicable institutional and governmental regulations concerning the ethical use of human volunteers were followed during the course of this research.

\section{References}

1. Jensen MP, Molton IR, Groah SL, Campbell ML, Charlifue S, Chiodo A, et al. Secondary health conditions in individuals aging with SCI: terminology, concepts and analytic approaches. Spinal Cord. 2012;50:373-8.

2. Jensen MP, Truitt AR, Schomer KG, Yorkston KM, Baylor C, Molton IR. Frequency and age effects of secondary health conditions in individuals with spinal cord injury: a scoping review. Spinal Cord. 2013;51:882-92.

3. Saunders LL, Clarke A, Tate DG, Forchheimer M, Krause JS. Lifetime prevalence of chronic health conditions among persons with spinal cord injury. Arch Phys Med Rehabil. 2015;96:673-9.

4. Rimmer JH, Chen MD, Hsieh K. A conceptual model for identifying, preventing, and managing secondary conditions in people with disabilities. Phys Ther. 2011;91:1728-39.

5. Goodman RA, Posner SF, Huang ES, Parekh AK, Koh HK. Defining and measuring chronic conditions: imperatives for research, policy, program, and practice. Prev Chronic Dis. 2013;10:120239.

6. US Department of Health and Human Services. Multiple chronic conditions - a strategic framework: optimum health and quality of life for individuals with multiple chronic conditions. Washington, DC; 2010. https://www.hhs.gov/sites/default/files/ash/initiatives/ mcc/mcc_framework.pdf

7. Hitzig SL, Eng JJ, Miller WC, Sakakibara BM. An evidencebased review of aging of the body systems following spinal cord injury. Spinal Cord. 2011;49:684-701.

8. Kemp B, Thompson L. Aging and spinal cord injury: medical, functional, and psychosocial changes. SCI Nurs. 2002; 19:51-60.

9. Cragg JJ, Noonan VK, Krassioukov A, Borisoff J. Cardiovascular disease and spinal cord injury: results from a national population health survey. Neurology. 2013;81:723-8.

10. LaVela SL, Evans CT, Prohaska TR, Miskevics S, Ganesh SP, Weaver FM. Males aging with a spinal cord injury: prevalence of cardiovascular and metabolic conditions. Arch Phys Med Rehabil. 2012;93:90-5.

11. Myers J, Lee M, Kiralti J. Cardiovascular disease in spinal cord injury: an overview of prevalence, risk, evaluation, and management. Am J Phys Med Rehabil. 2007;86:142-52.

12. Wahman K, Nash MS, Lewis JE, Seiger A, Levi R. Increased cardiovascular disease risk in Swedish persons with paraplegia: the Stockholm spinal cord injury study. J Rehabil Med. 2010;42:489-92.

13. Yang TY, Chen HJ, Sung FC, Kao CH. The association between spinal cord injury and acute myocardial infarction in a nationwide population-based cohort study. Spine. 2015;40:147-52.

14. Wu JC, Chen YC, Liu L, Chen TJ, Huang WC, Cheng H, et al. Increased risk of stroke after spinal cord injury: a nationwide 4year follow-up cohort study. Neurology. 2012;78:1051-7.

15. Cragg JJ, Noonan VK, Dvorak M, Krassioukov A, Mancini GB, Borisoff JF. Spinal cord injury and type 2 diabetes: results from a population health survey. Neurology. 2013;81:1864-8.
16. Lavela SL, Weaver FM, Goldstein B, Chen K, Miskevics S, Rajan $\mathrm{S}$, et al. Diabetes mellitus in individuals with spinal cord injury or disorder. J Spinal Cord Med. 2006;29:387-95.

17. Selassie A, Snipe L, Focht KL, Welldaregay W. Baseline prevalence of heart diseases, hypertension, diabetes, and obesity in persons with acute traumatic spinal cord injury: potential threats in the recovery trajectory. Top Spinal Cord Inj Rehabil. 2013;19:172-82.

18. Smith AE, Molton IR, Jensen MP. Self-reported incidence and age of onset of chronic comorbid medical conditions in adults aging with long-term physical disability. Disabili Hlth J. 2016;9:533-8.

19. Skinner HG, Coffey R, Jones J, Heslin KC, Moy E. The effects of multiple chronic conditions on hospitalization costs and utilization for ambulatory care sensitive conditions in the United States: a nationally representative cross-sectional study. BMC Health Serv Res. 2016;16:77.

20. Steiner CA, Barrett ML, Weiss AJ, Andrews RM. Trends and projections in hospital stays for adults with multiple chronic conditions, (MCC) 2003-2014: HCUP Statistical Brief \#183. Rockville, MD: Agency for Healthcare Research and Quality; 2014.

21. Lee TA, Shields AE, Vogeli C, Gibson TB, Woong-Sohn M, Marder WD, et al. Mortality rate in veterans with multiple chronic conditions. J Gen Intern Med. 2007;22(Suppl 3):403-7.

22. Vogeli C, Shields AE, Lee TA, Gibson TB, Marder WD, Weiss $\mathrm{KB}$, et al. Multiple chronic conditions: prevalence, health consequences, and implications for quality, care management, and costs. J Gen Intern Med. 2007;22(Suppl 3):391-5.

23. Lehnert T, Heider D, Leicht H, Heinrich S, Corrieri S, Luppa M, et al. Review: health care utilization and costs of elderly persons with multiple chronic conditions. Med Care Res Rev. 2011;68:387-420.

24. Parekh AK, Goodman RA, Gordon C, Koh HK. Conditions HHSIWoMC. Managing multiple chronic conditions: a strategic framework for improving health outcomes and quality of life. Public Health Rep. 2011;126:460-71.

25. Cardenas D, Hoffman J, Kirshblum S, McKinley W. Etiology and incidence of rehospitalization after traumatic spinal cord injury: a multicenter analysis. Arch Phys Med Rehabil. 2004;85:1757-63.

26. French DD, Campbell RR, Sabharwal S, Nelson AL, Palacios PA, Gavin-Dreschnack D. Health care costs for patients with chronic spinal cord injury in the Veterans Health Administration. J Spinal Cord Med. 2007;30:477-81.

27. DeVivo M, Farris V. Causes and costs of unplanned hospitalizations among persons with spinal cord injury. Top Spinal Cord Inj Rehabil. 2011;16:53-61.

28. Saunders LL, Murday D, Corley B, Cao Y, Krause JS. A comparison of rates of hospitalization and emergency department visits using self-report and South Carolina administrative billing data among a population-based cohort with spinal cord injury. Arch Phys Med Rehabil. 2016;97:1481-1486.

29. DeJong G, Tian W, Hsieh CH, Junn C, Karam C, Ballard PH, et al. Rehospitalization in the first year of traumatic spinal cord injury after discharge from medical rehabilitation. Arch Phys Med Rehabil. 2013;94(4 Suppl):S87-97.

30. Noonan VK, Fallah N, Park SE, Dumont FS, Leblond J, Cobb J, et al. Health care utilization in persons with traumatic spinal cord injury: the importance of multimorbidity and the impact on patient outcomes. Top Spinal Cord Inj Rehabil. 2014;20:289-301.

31. Mahmoudi E, Meade MA, Forchheimer MB, Fyffe DC, Krause JS, Tate D. Longitudinal analysis of hospitalization after spinal cord injury: variation based on race and ethnicity. Arch Phys Med Rehabil. 2014;95:2158-66.

32. January AM, Zebracki K, Czworniak A, Chlan KM, Vogel LC. Predictive factors of hospitalization in adults with pediatric-onset SCI: a longitudinal analysis. Spinal Cord. 2015;53:314-9. 
33. Gabbe BJ, Nunn A. Profile and costs of secondary conditions resulting in emergency department presentations and readmission to hospital following traumatic spinal cord injury. Injury. 2016;47:1847-55.

34. DeVivo M, Farris V. Causes and costs of unplanned hospitalizations among persons with spinal cord injury. Top Spinal Cord Inj Rehabil. 2011;16:53-61.

35. Dryden DM, Saunders LD, Rowe BH, May LA, Yiannakoulias N, Svenson LW, et al. Utilization of health services following spinal cord injury: a 6-year follow-up study. Spinal Cord. 2004;42:513-25.

36. Noreau L, Proulx P, Gagnon L, Drolet M, Laramee MT. Secondary impairments after spinal cord injury: a population-based study. Am J Phys Med Rehabil. 2000;79:526-35.

37. Savic G, Short DJ, Weitzenkamp D, Charlifue S, Gardner BP. Hospital readmissions in people with chronic spinal cord injury. Spinal Cord. 2000;38:371-7.
38. Middleton JW, Lim K, Taylor L, Sodden R, Rutkowski S. Patterns of morbidity and rehospitalisation following spinal cord injury. Spinal Cord. 2004;42:359-67.

39. Gerteis J, Izrael D, Deitz D, LeRoy L, Ricciardi R, Miller T, et al. Multiple chronic conditions chartbook. AHRQ Publications No., Q14-0038. Rockville, MD: Agency for Healthcare Research and Quality; 2014.

40. CDC. BRFSS Prevalence \& Trends Data [online] 2015. Available from: https://www.cdc.gov/brfss/brfssprevalence/.

41. NSCISC. Spinal cord injury facts and figures at a glance. Birmingham, AL: University of Alabama; 2017.

42. Krause JS, Saunders LL. Risk of hospitalizations after spinal cord injury: relationship with biographical, injury, educational, and behavioral factors. Spinal Cord. 2009;47:692-7.

43. Wolff JL, Starfield B, Anderson G. Prevalence, expenditures, and complications of multiple chronic conditions in the elderly. Arch Intern Med. 2002;162:2269-76. 